Manuelle Medizin 2008 • 46:384-385

DOI 10.1007/s00337-008-0652-z

Online publiziert: 6. Dezember 208

๑) Springer Medizin Verlag 2008
G. Plato, Rendsburg

\section{Der Weg zur Chronifizierung der kraniomandibulären Dysfunktionen (CMD)}

\section{Die Sicht des Orthopäden/Manualtherapeuten}

Kraniomandibuläre Dysfunktionen sind nur in ihren Anfangsstadien sicher und erfolgreich behandelbar. Bleiben diese Funktionsstörungen längere Zeit unentdeckt und unbehandelt, so beeinflussen sie nicht nur Nachbarsysteme, sondern haben nach und nach Funktionsstörungen im ganzen Körper zur Folge: Aus einer „lokalen“ Funktionsstörung entwickelt sich eine chronische Erkrankung.

An der Funktion eines Gelenkes sind beteiligt:

- Anteile der Materie/Struktur (Knorpelbandapparat, Kapsel, Diskus),

- Anteile der Energie (Muskeln, Bindegewebe, Faszien, Gefäße),

- Anteile der Steuerung (zentrales Nervensystem, periphere Nerven, autonomes Nervensystem).

Diese verschiedenen Anteile beeinflussen sich gegenseitig, Veränderungen an einem Anteil führen zwangsläufig zu Änderungen in den anderen Anteilen. Dies ist sowohl diagnostisch als auch therapeutisch nutzbar.

\section{Von der Interdependenz zum Regelkreis}

1. Im Regelkreisgeschehen kann jede äußere oder innere Störung zunächst kompensiert werden.

2. Bestehende Dysfunktionen müssen daher zunächst vom Patienten - bei ausreichender Kompensationsmöglichkeit im System - noch nicht bemerkt werden. Sie können aber von einem erfahrenen Untersucher bereits (oft) schon diagnostiziert werden. Ist die Kompensationsfähigkeit jedoch erschöpft (Zeitfaktor, weitere hinzukommende Störfaktoren), so werden Symptome auftreten, die nicht nur der Untersucher feststellen, sondern die jetzt auch der Patient deutlich benennen kann.

3. Die schwächsten Anteile bestimmen die Art und den Ort des Schmerzes.

Das heißt, wenn eine Dekompensation im kraniomandibulären System (CMS) vorliegt, muss sie nicht im Kopfbereich wehtun, sondern sie kann sich z. B. als Schmerz im Bereich der ersten und zweiten Rippe darstellen.

\section{- Der stärkste Schmerz muss nicht das führende Symptom sein.}

Dysfunktionen wird man also zunächst im Bereich des Arthrons erwarten können. Setzen Diagnostik und Therapie früh genug ein, werden die Funktionsstörungen auf das Arthron beschränkt bleiben.

Die Therapie wird sich auf die Fehlfunktion im Arthron, die Besserung der Funktion und die Behebung der Schmerzsymptomatik beschränken können.

\section{Das Arthron Kiefergelenk}

Werden Dysfunktionen im Bereich des Arthrons Kiefergelenk erwartet, müssen die drei Anteile der Funktion - 1. Materie/ Struktur, 2. Energie, 3. Steuerung - systematisch untersucht werden, um eine entsprechende Therapie einleiten zu können.
Ad 1: Um Probleme im Bereich der Materie zu überprüfen, müssen folgende Strukturen untersucht werden:

- die Gelenkflächen,

- der Diskus,

- der Bandapparat,

- die bilaminäre Zone.

Des Weiteren werden beurteilt:

- Veränderungen des Hart-/Weichgewebes in der Mundhöhle,

- Zahnstellung und Okklusion,

- aktive Bewegung und deren passive Weiterführung,

- Mundöffnung, Mundschluss, Seitwärtsbewegung, Vor- und Rückgleiten des Unterkiefers,

- Geräusche des Kiefergelenkes.

Ad 2: Um Probleme im Bereich der Energie zu überprüfen, müssen die folgenden Strukturen untersucht werden:

- die Muskulatur,

- die Faszien.

Sie werden beurteilt über:

- aktive Bewegung und deren passive Weiterführung,

- Endgefühl,

- Widerstandstests (isometrische Provokation),

- Prüfung der Kraft.

Ad 3: Um Probleme im Bereich der neuralen Steuerung auszuschließen, muss untersucht werden:

- die Funktion der Hirnnerven. 


\section{Dysfunktionen im Bereich des Musters}

Die Kompensation im Arthron ist erschöpft, wenn eine Adaptation im Arthron aus folgenden Gründen nicht mehr erfolgen kann:

1. Schwere der Schädigung der anatomischen bzw. funktionellen Strukturen,

2. hinzutretende Störmechanismen oder

3. Zeitfaktor (lang dauerndes Bestehen der Symptomatik der Funktionsstörung).

Es kommt zur Dekompensation. Dadurch werden Fehlfunktionen über die physiologischen Verbindungen des Arthrons an seine Nachbarn weitergegeben, die dort ebenfalls Fehlfunktionen auslösen. Diese Weiterleitung kann sowohl über Faszien, Muskeln, Nerven als auch über Gefäße erfolgen. Wir sprechen dann nicht mehr von einer Dysfunktion im Arthron, sondern von einer Dysfunktion im Bereich des Musters.

Bei Ausbreitung der Dysfunktion im Rahmen des Musters sind beim Betroffensein des Arthrons Kiefergelenk Störungen in folgenden Bereichen zu erwarten:

- in den Kopfgelenken (z. B. via Schädelbasis),

- in der übrigen Halswirbelsäule (via Faszien, Muskeln, nervale Verschaltung),

- im Mundboden (via nervale Verschaltung, mediale Faszie, Zunge, Hyoid, Muskeln),

- im Bereich des Hyoids (via Faszien, Muskeln),

- in den Halsfaszien (via Mandibula, nervale Verschaltung, Muskulatur),

- in der oberen Thoraxapertur mit den Rippen 1 und 2 (via Muskulatur, Faszien) und

- in der oberen Brustwirbelsäule bis $\mathrm{T}_{3}$ und $\mathrm{T}_{4}$ (via Muskulatur, Faszien).

Nach diesen Fehlfunktionen im Muster sollte gesucht werden, da bei diesen $\mathrm{Pa}$ tienten die Therapien nur im Bereich des Arthrons als nicht ausreichend anzusehen sind. Eine den jetzt bestehenden Dysfunktionsmustern entsprechende Ausweitung der Therapie ist notwendig, andernfalls ist der Weg zur weiteren Chronifizierung gebahnt. Eine interdisziplinäre simultane therapeutische Zusammenarbeit ist gefragt.

Verdächtig auf Dysfunktionen im Muster sind Schmerzsyndrome wie:

- Globusgefühl,

- Heiserkeit, Dysphonie,

- Schmerzen im Bereich des Mundbodens, der Zunge, des Zungengrundes und

- atypischer Gesichtsschmerz.

Bestehen bei Patienten neben den Dysfunktionen im CMS auch Beschwerden dieser Art, so sollte ein versierter Manualtherapeut hinzugezogen werden.

\section{Dysfunktionen im Bereich der Verkettung}

Unter dem Zeitfaktor - und bei fortschreitender Dekompensation - werden weitere Organe und Organsysteme von der Dysfunktion erfasst werden. Diese können oft anatomisch weit ab vom primären pathogenetischen Geschehen auftreten, sodass die Zusammenhänge (oft) nur schwer zu erkennen sind. Es handelt sich dann um Dysfunktionen im Bereich der Verkettung.

Die folgenden Strukturen sind besonders häufig bei der Verkettungssymptomatik an Fehlfunktionen beteiligt: die substernalen Faszien, das Diaphragma abdominalis, die Viszera, der Beckenboden, das Becken, das Coccyx, die übrige Wirbelsäule.

Bestehen neben der kraniomandibulären Dysfunktion auch noch Beschwerden wie Zephalgie, Schwindel, Tinnitus, Atemnotsyndrome, funktionelle Herzbeschwerden, Darmstörungen oder Störungen des Beckenbodens, so sollte interdisziplinäre Hilfe angefordert werden. Nach Ausschluss eines anderen pathologischen Geschehens als den Funktionsstörungen sollten die Dysfunktionen manualtherapeutisch/osteopathisch unbedingt behandelt werden. Eine Behandlung nur der CMD würde mit hoher Wahrscheinlichkeit keinen ausreichenden und bleibenden Erfolg bringen.

\section{Korrespondenzadresse}

Dr. G. Plato

Holstenstraße 4

24768 Rendsburg

gernotplato@t-online.de
Bildschirmarbeit in Kliniken Mängel in der Gestaltung von Arbeitsplätzen müssen behoben werden

Von der Diagnose durch Tomographie oder Ultraschall über computergestützte Operationstechniken bis hin zur Pflege und Verwaltung: Medizin ist ohne Computer nicht mehr denkbar. Die Entwicklung ständig neuer Medizintechnologie hat die Lebens- und Behandlungsqualität der Patienten erheblich verbessert. Doch für das Personal, das Bildschirmarbeit an Großgeräten ausübt, bedeutet dies eine zusätzliche Belastung.

Ein Forschungsprojekt im Auftrag der Bundesanstalt für Arbeitsschutz und Arbeitsmedizin (BAuA) untersuchte 130 Bildschirmarbeitsplätze an medizinischen Großgeräten wie Röntgen-, Ultraschall-, EKG- oder EEG-Geräten in 14 Krankenhäusern. Die Studie ergab, dass Faktoren wie ein zu hoher Geräuschpegel, zu trockene Luft, mangelhafte Beleuchtung sowie Belastungen durch die Arbeitsorganisation die Leistungsfähigkeit und das Wohlbefinden der Angestellten beeinträchtigen. Hier hat der technische Fortschritt die Arbeitsplatzgestaltung überholt. Der vor kurzem veröffentliche Bericht „Bildschirmarbeit in Kliniken und Praxen" der BAuA gibt Hinweise zur Verbesserung und Optimierung solcher Bildschirmarbeitsplätze durch simple Maßnahmen wie bessere Beleuchtung, einen veränderten Standort oder mehr Arbeitsfläche.

Weitere Informationen unter: www.baua.de/de/Publikationen/ Fachbeitraege/F1801.html

Quelle: Bundesanstalt für Arbeitsschutz und Arbeitsmedizin 